\title{
Estimation and validation of hybrid choice models to identify the role of perception in the choice to cycle
}

\author{
Eleonora Sottile $^{\mathrm{a}^{*}}$, Benedetta Sanjust di Teulada $^{\mathrm{a}}$, Italo Meloni ${ }^{\mathrm{a}}$, Elisabetta Cherchi ${ }^{\mathrm{b}}$ \\ ${ }^{a}$ University of Cagliari, Italy, CRIMM, via San Giorgio 12, 19124 Cagliari \\ ${ }^{b}$ Newcastle University, United Kingdom, NE1 7RU
}

\begin{abstract}
Cycling is one of the most sustainable and ecofriendly modes of travel and a good form of exercise. Many government and public health authorities recommend cycling to stay fit as well as to reduce air and noise pollution, $\mathrm{CO}_{2}$ emissions, traffic congestion and other negative consequences of car use. In light of these benefits, a major challenge for researchers today is how to promote cycling. However, in countries where cycling is not common, apart from the need for proper cycling facilities, one major issue concerns people's perception of cycling for sport or recreational activities rather than as a mode of transport. The aim of this paper is to explore the role of perception in the likelihood of the bike being used for utilitarian purposes. We focus on the perception of: the bicycle as a means of transport; bikeability (in terms of usefulness and safety) and of bike infrastructure. Hybrid Choice Models (HCM) have been used to estimate the effect of people's perception on the propensity to bike. The HCM also accounts for the serial correlation between error terms in the discrete and latent perceptions, to allow for agent-common unknown factors. Further, we also validate the model results using a hold-out sample and discuss some policy measures aimed at changing travel behaviour. The results suggest that, besides individual characteristics, latent aspects related to the perception of the context and of the bicycle as a means of transport strongly affect the propensity to cycle.
\end{abstract}

Keywords: choice of cycling; hybrid choice model; validation; simulation; serial correlation

\footnotetext{
${ }^{*}$ Corresponding author. Tel.: +390706756405; fax: +390706756401 .
}

E-mail address: elesottile@gmail.com 


\section{Introduction}

The promotion of cycling can help to abate environmental and traffic-related problems caused by motorised forms of transport. Considering the benefits of cycling, a major challenge for researchers today is how to encourage this form of transportation. Denmark and The Netherlands are famous for their cycling culture (Carstensen and Ebert, 2012), However, in the majority of other European countries cycling mode share is very low: only $2 \%$ of trips are made by bicycle in Great Britain 3\% in Ireland and the Czech Republic and 5\% in France (Van Hout Kurt, 2008), figures confirmed also at the Capitals level $\left(\mathrm{ECF}^{\dagger}\right)$. In Italy, though $50 \%$ of daily trips do not cover more than $5 \mathrm{~km}$, cycling is the least-used mode of transportation, accounting for just $3.8 \%$ of daily trips (Isfort, 2014 $4^{\ddagger}$ ). In countries with low cycling mode share, bicycles are used above all for sport and recreational activities. The lack of proper infrastructure has precluded the development of a cycling culture, strengthening the perception of the bicycle as a form of recreation (in urban areas not accessible to motor vehicles) rather than as a mode of transport. Thus, in order to increase the propensity to cycle, user perception needs to be changed. In addition to providing proper cycling facilities, it is important to evaluate individuals' perception of bikes as an alternative mode of travel.

Promoting bicycle use requires understanding those factors underpinning the propensity to cycle and also the structural and psychosocial barriers that may contribute to hindering use of the bike (Pucher et al., 2010; Heinen et al., 2010, Fernàndez-Heredia et al., 2014). There exists a broad literature on the objective factors (context characteristics, facilities, socio-economic and demographic factors) affecting the propensity to cycle (see for example Kingham et al., 2001; Dickinson et al., 2003; Pucher and Buehler, 2006; Dill and Voros, 2007; Hunt and Abraham, 2007; Parkin et al., 2008; Heinen et al., 2010; Vandenbulcke et al., 2011; Broach et al., 2012; Krizek, 2012; Calvey et al., 2015) as well as on the psycho-social factors. For example, positive attitudes toward cycling (Li et al., 2013), (Willis et al., 2015) convenience (flexible, efficient) and exogenous restrictions (danger, vandalism, facilities), (FernàndezHeredia et al., 2014) are indicated as having a positive effect on the choice to cycle. Social norms explain why cycling in some areas in Northern Europe is particularly common (Pucher et al., 1999; Wardman et al., 2007). The importance that individuals attach to the health benefits of cycling for example also has an effect on cycling; the same goes for environmental beliefs (Gatersleben and Appleton, 2007; Heinen et al., 2011; Hunecke et al., 2001; Pooley et al., 2012; Emond and Handy, 2012). Dill \& Voros (2007) found that social norms affect cycling behaviour: people living in households with other adults who cycle regularly, had co-workers who cycled to work, or who frequently saw adults cycling on their street were more likely to be regular cyclists themselves. According to Stinson \& Bhat (2005) cycling more in leisure time could increase the frequency of bicycle use for commuting.

Among the psycho-social factors, individuals' perception of the system plays an important role in biking.

\footnotetext{
${ }^{\dagger}$ https://ecf.com/resources/cycling-facts-and-figures

thttp://www.isfort.it/sito/statistiche/Congiunturali/Annuali/RA_2014.pdf, in Italian.
} 
Gatersleben and Appleton (2007) observed that non-cyclists perceive more barriers to cycling than utility cyclists and vice versa (Bamberg and Schmidt, 1994). Majumdar and Mitra (2015) studied several perceived benefits (physical fitness, environmental awareness, travel reliability, travel flexibility, psychological safety, affordability, desire for pollution free roads) and found safety hazards, social barriers and road conditions to influence the choice to travel by bike the most. A comparative study of cyclists in Brisbane and Copenhagen on the perceptions of safety (Chataway et al. 2014) showed that cyclists in Brisbane perceived mixed traffic environments as less safe, and felt more apprehensive of traffic than cyclists in Copenhagen. Ma et al. (2014) explored the relationships between the objectively measured environment, perceptions of the environment, and cycling behaviour. The results of their study showed that the perception of the environment had a direct and significant effect on cycling behaviour, while the direct effect of the objective environment on cycling behaviour became insignificant when controlling for perception. They concluded that a good cycling environment was necessary but not sufficient for using the bike. Akar and Clifton (2009) explored the perceptions of the campus community regarding possible cycling infrastructure improvements, policy, and programme innovations. Kaplan et al. (2015) investigated the behavioural factors underlying tourist intentions to use urban bike-sharing for recreational cycling while on holiday. Sigurdardottir et al. (2013) focused on the intentions of adolescents to commute by car or bicycle as adults. Muñoz et al. (2016) proposed a methodology for including cycling-related indicators in mobility surveys based on the theory of planned behaviour.

None of these works studies the perception of the bike as a mode of transport. They also use factor analyses alone or in conjunction with structural equation models to study the relationship between psycho-social factors and biking.

Only a few applications of Hybrid Choice Models (HCM) for estimating the effect of psycho-social factors on the choice to cycle are reported in the literature. Kamargianni and Polydoropoulou (2013) estimated the influence of the latent variable willingness to walk or cycle on mode choice. Motoaki and Daziano (2015) investigated the effects of weather (temperature, rain, and snow), cycling time, slope, cycle facilities (bike lanes), and traffic on cycling decisions. Maldonado-Hinarejos et al. (2014) incorporated attitudes towards cycling, perceptions of the image associated with cycling and the stress arising from safety concerns in a choice model for cycling. La Paix et al. (2015) estimated the impact of the perception of the quality of bicycle interchanges and attitudes towards cycling on the mode choice (including the bike) to access/egress rail stations. Habib et al. (2014) studied the effect of comfort, safety consciousness and perceptions of bikeability (in terms of quality of cycling facilities), on the choice of biking for utilitarian or recreational purposes.

This paper aims specifically to explore the role of perception in the likelihood of utility cycling. We focus on the perception of: the bicycle as a means of transport; bikeability (in terms of usefulness and safety) and of bike infrastructure. Hybrid Choice Models (HCM) have been used to estimate the effect of people's perception on the propensity to bike. Unlike all earlier works, our HCM also accounts for the serial correlation between error terms in the discrete and latent perceptions, to allow for agent-common 
unknown factors that equally affect both the discrete choice to use the bike and the latent perceptions of it. We also use revealed preference instead of stated preference data, to represent the actual context. In this way, we can capture individuals' current perceptions so as to define the correct measures to be adopted for increasing the propensity to cycle. Further, we also validate the model results, using a holdout sample. The validation phase is crucial and highly recommended for assessing the quality of the models estimated, but it has been widely neglected in transportation research. Few exceptions are Cherchi and Cirillo (2010), Mabit et al. (2015) and Klapper et al. (2005).

The remainder of the paper is structured as follows: Section 2 describes the methodology adopted. Section 3 presents the application and provides a descriptive analysis of the dataset including both the objective characteristics and perceptions. Section 4 discusses the modelling framework and Section 5 the model results. Section 6 concludes the paper.

\section{Methodological framework}

The methodology followed envisaged a preliminary phase for survey design. The survey aimed, on the one hand, to gather information on current travel habits focusing on bicycle mode, and on the other to identify and measure (using a Likert scale) those factors underpinning the choice to use/not to use the bike. We focused in particular on analysing how people perceive the bicycle and related aspects (safety, infrastructure, etc.). We then performed a confirmatory factor analysis on the survey data so as to pinpoint which of the items contained in the questionnaire better represented the a priori assumption of perceptions. Lastly, and most importantly we analysed the effect of perceptions on the propensity to use/not to use the bicycle. We achieved this objective by estimating and validating a hybrid discrete choice model that allowed us to identify those socio-economic characteristics influencing to the greatest extent the propensity to cycle/not to cycle and at the same time the role played by perceptions on that propensity.

\section{Application}

The data used in the analysis were drawn from a web-survey, called "Bicimipiaci" ("Bikellikeyou"), conducted, between 2014 and 2016, by the University of Cagliari (Italy) in collaboration with public authorities, among a sample of Sardinian Regional government, University and municipal employees. E-mails were sent to around 9,600 individuals inviting them to participate in a web-survey. A number of prizes $\S$ were offered as an incentive to fill in the questionnaire. 4,691 individuals completed the survey, a very high response rate (48.9\%). Questionnaires were carefully analysed and 2,752 observations were used for modelling purposes (corresponding to $28.6 \%$ of the individuals contacted and to $58.6 \%$ of

\footnotetext{
$\S$ The lottery comprised: one bicycle, 15 one-year bike-sharing cards, 1 one-year car-sharing card, and various cycling gadgets.
} 
respondents). Clearly, the sample was not intended to be representative of the general population, but sample size was large enough to permit interesting analyses.

The questionnaire was divided into 4 sections. The first section separated out cyclists from non-cyclists and aimed to identify why and how the former chose to cycle for utilitarian purposes. The second section was designed to measure perceptions using the 5-point Likert scale, specifically 1) positive and negative perceptions about cycling in general, 2) the "perception of context characteristics", intended as the importance assigned to policies for increasing bicycle use, 3) the "perception of bikeability and safety" of bike lanes and paths and to detect the main barriers to cycling (non-cyclists). The third section consisted of the description of the daily commute. The last part was dedicated to gathering information about individuals socio-economic characteristics. (More details in Meloni et al., 2016).

The study context concerned small and medium sized towns which did not have a complete urban cycle system when the survey was conducted. The majority of existing cycle lanes had been created on the roadway between the parking lane and the pavement. The lack of a capillary network meant that it was not possible to design complete routes between a given origin and destination. A bike sharing service was introduced in the capital Cagliari in 2010, initially with 4 stations which were increased to 10 in 2013 for a total of 100 bikes, of which 30 pedal assisted. However, by February 2015 this service was no longer accessible.

\subsection{Data analysis}

The final sample used for the estimation comprises the same proportion of non-cyclists $(50.1 \%)$, and frequent cyclists (49.9\%). Despite making up half the sample, only $20.7 \%$ of cyclists use the bike several times a week or every day. Four different purposes for cycling are investigated: commuting, shopping, leisure and travelling to a public transport stop. Frequency of biking was measured with a 5-level scale: 1) never, 2) 1-10 times a year, 3) 1-5 times per month, 4) several times a week, 5) every day. The frequency analysis confirms that the bicycle is used mostly for recreational purpose $(98.1 \%$ ), whereas $66.7 \%$ indicated "never" for cycling to work, and $88.3 \%$ for travelling to a public transport stop. This is not surprising since in the context under study the culture of utility cycling is inexistent; the bike is used for leisure.

Turning to individual and household characteristics, Table 1 shows that the sample is practically equally divided between males and females. Average age is 48 years, though $74.6 \%$ of the sample are aged between 41 and 60. As expected, the sample is relatively educated, and the majority are wage earners. The largest proportion have a monthly income of between $€ 1,000 \div 2,000$, are married with children and the average number of household members is around three. $97.6 \%$ of the respondents have a driving licence and $90.5 \%$ are car owners. The average number of cars is higher than the average number of bicycles per household, though interestingly surprisingly high, considering that cycling is not common in 
Italy. The sample was divided into two subsamples, one for model estimation ("Estimation sample") and the other for validation ("Validation sample"). The validation sample includes 550 observations and was obtained by random sampling $20 \%$ of observations from the total. The remaining $80 \%$ (corresponding to 2,202 observations) was used for model estimation. Table 1 shows the socio-economic characteristics for both the estimation and validation subsamples which, as can be seen, do not differ significantly.

Table 1: Socioeconomic characteristics

\begin{tabular}{|c|c|c|c|c|c|c|c|c|c|}
\hline \multirow{2}{*}{\begin{tabular}{|c|}
$\begin{array}{r}\text { Socio-Economic } \\
\text { Characteristics }\end{array}$ \\
Variables \\
\end{tabular}} & \multicolumn{3}{|c|}{$\begin{array}{c}\text { Total Sample } \\
(2,752 \text { obs. }) \\
\end{array}$} & \multicolumn{3}{|c|}{$\begin{array}{c}\text { Estimation Sample } \\
(2,202 \text { obs. }) \\
\end{array}$} & \multicolumn{3}{|c|}{$\begin{array}{c}\begin{array}{c}\text { Validation Sample } \\
(550 \text { obs. })\end{array} \\
\end{array}$} \\
\hline & $\overline{\text { AVG }}$ & No. & $\%$ & AVG & No. & $\%$ & AVG & No. & $\%$ \\
\hline Bicycle use & & 1,373 & 49.9 & & 1,095 & 49.73 & & 278 & 50.55 \\
\hline $\begin{array}{l}\text { Age } \\
\text { Age_18-30 } \\
\text { Age_31-40 } \\
\text { Age_41-60 } \\
\text { Age }>60 \\
\end{array}$ & 48.00 & $\begin{array}{c}104 \\
458 \\
2,004 \\
186 \\
\end{array}$ & $\begin{array}{c}3.78 \\
16.64 \\
72.82 \\
6.76 \\
\end{array}$ & 47.93 & $\begin{array}{c}87 \\
370 \\
1,603 \\
142 \\
\end{array}$ & $\begin{array}{c}3.95 \\
16.80 \\
72.80 \\
6.45 \\
\end{array}$ & 48.30 & $\begin{array}{c}17 \\
88 \\
401 \\
44 \\
\end{array}$ & $\begin{array}{c}3.09 \\
16.00 \\
72.91 \\
8.00 \\
\end{array}$ \\
\hline Gender: male & & 1,399 & 50.84 & & 1,129 & 51.27 & & 270 & 49.09 \\
\hline $\begin{array}{l}\text { Level of Education } \\
\text { Middle school or lower } \\
\text { High school } \\
\text { Specialization } \\
\text { Undergraduate and master's degree } \\
\text { Post lauream (phd, etc.) }\end{array}$ & & \begin{tabular}{|c|}
113 \\
1,042 \\
63 \\
915 \\
619 \\
\end{tabular} & $\begin{array}{c}4.11 \\
37.86 \\
2.29 \\
33.25 \\
22.49 \\
\end{array}$ & & $\begin{array}{c}96 \\
813 \\
49 \\
733 \\
511 \\
\end{array}$ & $\begin{array}{c}4.36 \\
36.92 \\
2.23 \\
33.29 \\
23.21 \\
\end{array}$ & & \begin{tabular}{|c|}
17 \\
229 \\
14 \\
182 \\
108 \\
\end{tabular} & $\begin{array}{c}3.09 \\
41.64 \\
2.55 \\
33.09 \\
19.64 \\
\end{array}$ \\
\hline $\begin{array}{l}\text { Marital status: married } \\
\text { With children } \\
\# \text { of members in the household }\end{array}$ & 2.88 & $\begin{array}{l}2,007 \\
1,517\end{array}$ & $\begin{array}{l}72.93 \\
55.12\end{array}$ & 2.88 & $\begin{array}{l}1,601 \\
1,214\end{array}$ & $\begin{array}{l}72.71 \\
55.13\end{array}$ & 2.89 & $\begin{array}{l}406 \\
303\end{array}$ & $\begin{array}{l}73.82 \\
55.09\end{array}$ \\
\hline $\begin{array}{l}\text { Driving licence } \\
\text { Personal car available } \\
\text { \#of cars in the household } \\
\text { \#of bikes in the household }\end{array}$ & $\begin{array}{l}1.71 \\
1.53 \\
\end{array}$ & $\begin{array}{l}2,688 \\
2,491\end{array}$ & $\begin{array}{l}97.67 \\
90.52\end{array}$ & $\begin{array}{l}1.71 \\
1.53 \\
\end{array}$ & $\begin{array}{l}2,158 \\
1,992\end{array}$ & $\begin{array}{l}98.00 \\
90.46\end{array}$ & $\begin{array}{l}1.70 \\
1.53 \\
\end{array}$ & $\begin{array}{l}530 \\
499\end{array}$ & $\begin{array}{l}96.36 \\
90.73\end{array}$ \\
\hline $\begin{array}{l}\text { Not informed about bike sharing } \\
\text { Informed about bike sharing, non- } \\
\text { subscriber } \\
\text { Informed about bike sharing, subscriber } \\
\text { Informed about bike sharing }\end{array}$ & & \begin{tabular}{|c|}
1,009 \\
\\
1,349 \\
39 \\
355 \\
\end{tabular} & $\begin{array}{c}36.66 \\
49.02 \\
1.42 \\
12.90 \\
\end{array}$ & & $\begin{array}{c}786 \\
1,091 \\
28 \\
297 \\
\end{array}$ & $\begin{array}{c}35.69 \\
49.55 \\
1.27 \\
13.49 \\
\end{array}$ & & \begin{tabular}{|c|}
223 \\
258 \\
11 \\
58 \\
\end{tabular} & $\begin{array}{c}40.55 \\
\\
46.91 \\
2.00 \\
10.55 \\
\end{array}$ \\
\hline $\begin{array}{l}\text { No Income } \\
\text { Low level }(<1,000 €) \\
\text { Medium level }(1,000 €-2,000 €) \\
\text { Medium - High level }(2,000 €-3,000 €) \\
\text { High level }(>3,000 €)\end{array}$ & & \begin{tabular}{|c|}
49 \\
124 \\
1,823 \\
375 \\
381 \\
\end{tabular} & $\begin{array}{l}1.78 \\
4.51 \\
66.24 \\
13.63 \\
13.84 \\
\end{array}$ & & \begin{tabular}{c|}
38 \\
105 \\
1,445 \\
302 \\
312 \\
\end{tabular} & $\begin{array}{l}1.73 \\
4.77 \\
65.62 \\
13.71 \\
14.17 \\
\end{array}$ & & $\begin{array}{c}11 \\
19 \\
378 \\
73 \\
69 \\
\end{array}$ & $\begin{array}{l}2.00 \\
3.45 \\
68.73 \\
13.27 \\
12.55 \\
\end{array}$ \\
\hline
\end{tabular}




\subsection{Perceptions}

The latent variables have been defined on the basis of the items reported in Table 2. Each respondent was asked to express her/his level of agreement or disagreement with each item on a Likert scale from 1 to 5 ( $1=$ Totally disagree to $5=$ Totally agree). In particular, as mentioned, we focused on the three latent variables relating to: (1) perception of the bicycle as a means of transport, defined at the personal and societal level by twelve items; (2) perception of context characteristics, described by four items; (3) perception of bikeability, defined by nine items.

A first descriptive statistics analysis revealed that the sample perceived positively the bicycle as a means of transport, though cycling in traffic was considered dangerous and bikes unsuitable for carrying heavy items. As was to be expected, the perception of bikeability confirmed the dangers users associate with cycling in traffic. This could create a barrier to bike use, given the lack of adequate cycling infrastructure in the reference context. Added to this is the fact that existing bike lanes are not perceived as useful for travelling in urban areas and that motorists encroach on cyclists' road spaces.

As for the perception of context characteristics in the propensity to cycle, this was judged to be important, especially the presence of a dedicated bicycle network within urban areas, of bike racks and secure parking and extending restricted traffic zones or pedestrian zones. These are a necessary condition for the bicycle to be regarded as an alternative transport mode. Without proper infrastructure and facilities people are unlikely to consider the bicycle as an available travel mode option (see Table 2). However, the presence of appropriate cycling facilities is not the only condition for choosing to cycle. Indeed, individuals who do not recognise the attributes associated with bicycle use are less likely to choose to cycle.

We performed two types of factor analysis to identify the latent dimensions underpinning our set of items: Principal Component Analysis (PCA) and Factor Analysis (FA). To determine the factorability of the data and the number of factors to be extracted, we used the Kaiser-Meyer-Olkin (KMO) test (>0.5), the scree or Cattell test (Cattell, 1966) and parallel analysis. Tables 2 and 3 show the factor analysis results, in particular the first one shows the weight of each item for the first factor identified by the FA for the total sample, for the estimation sample (2,202 individuals) and for the validation sample (550 individuals), and the second one the factorability and the reliability of the three latent variables. However only two of the three latent variables were found to be significant: perception of the bicycle as a means of transport and perception of the context (defined both by the items underlined in the table) whereas perception of bikeability (in terms of usefulness and safety) was below the reliability threshold as shown in Table 3 . As will be seen later, the model estimation confirmed that this latent variable was indeed problematic. 
Table 2: Factor analysis results (weights for the first factor)

\begin{tabular}{|c|c|c|c|c|}
\hline \multicolumn{5}{|c|}{ Factor analysis results (weights for the first factor) } \\
\hline & & \begin{tabular}{|c|} 
Total \\
Sample \\
\end{tabular} & $\begin{array}{c}\text { Estimation } \\
\text { Sample }\end{array}$ & $\begin{array}{c}\text { Validation } \\
\text { Sample } \\
\end{array}$ \\
\hline Items (Indicators of Latent Variables) & Name & (2,752 obs.) & $(2,202$ obs. $)$ & (550 obs.) \\
\hline \multicolumn{5}{|l|}{ Latent variable: Perception of bicycle as a means of transport } \\
\hline It is a rapid means of transport (avoids queues and traffic) & Perc_1 & 0.417 & 0.451 & 0.343 \\
\hline Cycling in traffic is not dangerous & Perc_2 & 0.011 & -0.022 & 0.118 \\
\hline It is likely to be stolen and parking areas are inadequate & Perc_3r & 0.038 & 0.006 & -0.030 \\
\hline It is not expensive & Perc 4 & $\underline{0.574}$ & $\underline{0.537}$ & $\underline{0.717}$ \\
\hline It involves exposure to bad weather and air pollution & Perc_5r & 0.030 & 0.001 & 0.073 \\
\hline It avoids wasting time looking for parking & $\underline{\text { Perc } 6}$ & $\underline{0.580}$ & $\underline{0.557}$ & $\underline{0.645}$ \\
\hline It is healthy & Perc 7 & $\underline{0.776}$ & $\underline{0.767}$ & $\underline{0.754}$ \\
\hline It is difficult to carry heavy items & Perc_8r & -0.109 & -0.125 & -0.070 \\
\hline It allows one to appreciate historic centres and increases accessibility & & & & \\
\hline$\underline{\text { to city services }}$ & $\underline{\text { Perc } 9}$ & $\underline{0.691}$ & $\underline{0.689}$ & $\underline{0.699}$ \\
\hline Need for cycling gear & Perc_10r & -0.087 & -0.038 & -0.130 \\
\hline It contributes to reducing polluting emissions & $\underline{\text { Perc } 11}$ & $\underline{0.740}$ & $\underline{0.746}$ & $\underline{0.684}$ \\
\hline It limits daily activity patterns & Perc_12r & 0.041 & 0.104 & -0.067 \\
\hline \multicolumn{5}{|c|}{ Latent variable: Perception of bikeability (in terms of usefulness and safety) } \\
\hline Existing bike lanes are not useful for travelling & Bikeab 1 & $\underline{0.792}$ & $\underline{0.788}$ & $\underline{0.82}$ \\
\hline $\begin{array}{l}\text { Existing bike lanes and crossings are safe, comfortable and well- } \\
\text { marked }\end{array}$ & Bikeab 2r & 0.701 & 0.682 & 0.728 \\
\hline It is better to ride in traffic than use the existing bike paths & $\underline{\text { Bikeab } 3}$ & $\overline{0.582}$ & $\overline{0.609}$ & $\overline{0.548}$ \\
\hline Motorists often encroach on dedicated bike lanes & Bikeab_4 & 0.23 & 0.233 & 0.084 \\
\hline \multicolumn{5}{|c|}{ Latent variable: Perception of context characteristics in propensity to cycle } \\
\hline A dedicated cycle network in urban areas & Context 1 & $\underline{0.938}$ & $\underline{0.997}$ & $\underline{0.932}$ \\
\hline The presence of bike racks and secure parking & Context 2 & $\underline{0.953}$ & $\underline{0.962}$ & $\underline{0.939}$ \\
\hline Extending RTZ or pedestrian zones & Context_3 & $\overline{0.679}$ & $\overline{0.659}$ & $\overline{0.728}$ \\
\hline A bike-sharing station close to home or at public transport stops & Context_4 & 0.356 & 0.371 & 0.453 \\
\hline If other people use it & Context_5 & -0.211 & -0.203 & -0.138 \\
\hline $\begin{array}{l}\text { Dedicated facilities at work / study (parking, showers, lockers for } \\
\text { equipment, etc.) }\end{array}$ & Context_6 & 0.351 & 0.413 & 0.255 \\
\hline An integrated ticket for bike-sharing and public transport services & Context_7 & 0.162 & 0.255 & 0.046 \\
\hline Combination with public transport services & Context_8 & 0.209 & 0.318 & 0.018 \\
\hline Increase of parking fees & Context 9 & -0.241 & -0.258 & -0.158 \\
\hline
\end{tabular}

\footnotetext{
*the $\mathrm{r}$ means it was analysed in reverse.
} 
Table 3: Factorability and reliability

\begin{tabular}{|c|c|c|c|c|c|}
\hline Latent Variables & Sample & KMO & $\begin{array}{c}\text { No. Indicators factor } \\
1 \\
\end{array}$ & Alpha factor 1 & $\begin{array}{c}\% \text { of variance } \\
\text { explained }\end{array}$ \\
\hline \multirow{3}{*}{$\begin{array}{l}\text { Perception of bicycle as a } \\
\text { means of transport }\end{array}$} & Total Sample & 0.776 & \multirow{3}{*}{5} & 0.680 & 22.891 \\
\hline & Estimation Sample & 0.767 & & 0.724 & 22.660 \\
\hline & Validation Sample & 0.775 & & 0.724 & 24.133 \\
\hline \multirow{3}{*}{$\begin{array}{l}\text { Perception of bikeability } \\
\text { (in terms of usefulness } \\
\text { and safety) }\end{array}$} & Total Sample & 0.559 & \multirow{3}{*}{3} & 0.489 & 37.774 \\
\hline & Estimation Sample & 0.566 & & 0.486 & 37.761 \\
\hline & Validation Sample & 0.528 & & 0.486 & 38.011 \\
\hline \multirow{3}{*}{$\begin{array}{l}\text { Perception of context } \\
\text { characteristics in } \\
\text { propensity to cycle }\end{array}$} & Total Sample & 0.814 & \multirow{3}{*}{3} & 0.773 & 45.293 \\
\hline & Estimation Sample & 0.823 & & 0.776 & 46.042 \\
\hline & Validation Sample & 0.775 & & 0.776 & 42.363 \\
\hline
\end{tabular}

Figure 1 shows the distribution of responses for the estimation sample (2,202 individuals) for those items identifying the perception of the bicycle as a means of transport and for perception of the context.

It clearly emerges that the majority of this subsample (60 to 90\%) has a positive perception of the bicycle as a form of transport, recognizing the associated benefits. The same goes for perception of the context characteristics, though to a lesser extent, that are indeed encourage the propensity to cycle. 
Figure 1: Distribution of responses of the estimation sample

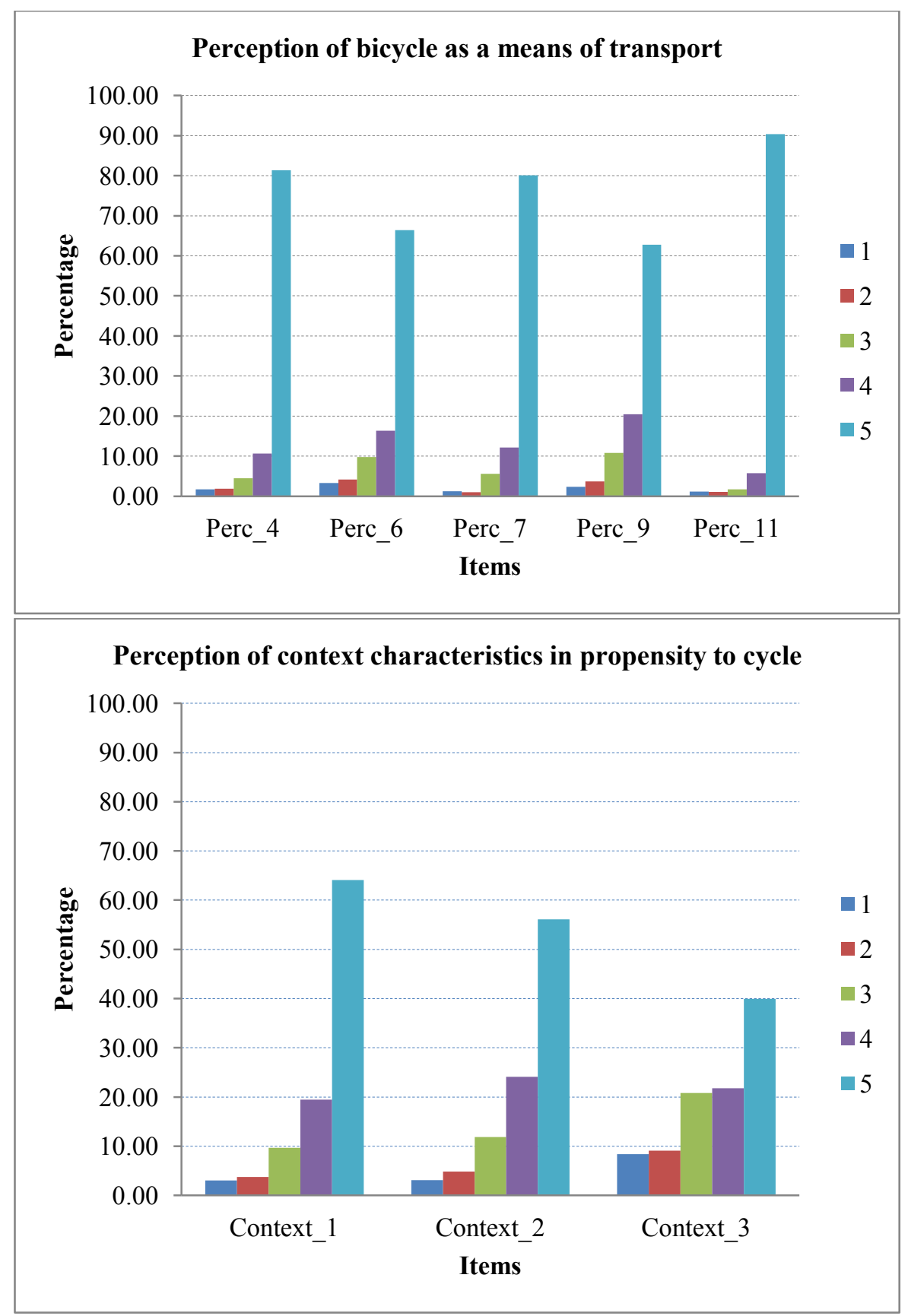




\section{Modelling framework}

The hybrid choice model used here is a binary logit that models the choice to cycle $v s$. the choice not to cycle as a function of a set of socioeconomic characteristics and three latent variables that measure individuals' perception of different aspects of biking. In addition, we also account for serial-correlation between the error terms. Since both the discrete and the latent part apply to the same individual making the choice and provided an evaluation of the indicators, the error terms of these sub-models may be correlated, as they potentially share unobserved variables specific to each individual. Following Bierlaire (2016) we deal with serial correlation by incorporating an agent effect in the model specification. This is an error component appearing in all the sub-models involved, distributed across the individuals. Let us denote with $\Delta U_{q}$ the difference between the utilities that the individual $q$ associates to the alternatives of cycling and not cycling:

$$
\Delta U_{q}=A S C+\boldsymbol{\beta} \cdot \boldsymbol{S} \boldsymbol{E}_{q}+\sum_{n}\left(\lambda_{n}+\boldsymbol{\theta}_{\boldsymbol{n}} \cdot \boldsymbol{S} \boldsymbol{E}_{q}\right)\left(C_{n}+\boldsymbol{\alpha}_{n} \cdot \boldsymbol{S} \boldsymbol{E}_{q}^{\prime}+\omega_{q n}+\eta_{q}\right)+\varepsilon_{q}+\eta_{q}
$$

where $\boldsymbol{S} \boldsymbol{E}_{\boldsymbol{q}}$ is a vector of socioeconomic characteristics, $\boldsymbol{\beta}$ the respective vector of coefficients, $A S C$ is the constant specific for the cycling alternative, and $L V_{n q}=C_{n}+\boldsymbol{\alpha}_{n} \cdot \boldsymbol{S} \boldsymbol{E}_{q}^{\prime}+\omega_{q n}+\eta_{q}$ is the $n$-th latent variable (with $n=1,2,3$ ) that depends on a vector of socioeconomic characteristics $\left(\boldsymbol{S} \boldsymbol{E}_{q}\right.$ '), which can be different from that included in the discrete choice, with $\boldsymbol{\alpha}_{n}$ the associated coefficients. $C_{n}$ is the intersect, $\omega_{q n}$ is a normally distributed error term with zero mean and standard deviation $\sigma_{\omega}$, and $\lambda_{n}$ is the coefficient associated with each LV. Lastly, $\boldsymbol{\theta}_{n}$ is a vector of coefficients associated with the interaction between LV and SE, while $\varepsilon_{q}$ is the logistically distributed error term and $\eta_{\mathrm{q}}$ is a normally distributed error component with zero mean and standard deviation $\sigma_{r}$, which is common between the LVs and the utility of the discrete alternatives.

The items reported in Table 2 are used as indicators of the latent variables and are related thereto by means of the following measurement equation:

$$
I_{q n k}=\gamma_{k}+\zeta_{k} L V_{q n}+v_{q k} \quad k=1, \ldots, K
$$

where $I_{q n k}$ is the $k$-th indicator for the $n$-th latent variable, $\gamma_{k}$ is the intersect, $\zeta_{k}$ is the coefficient associated with the latent variable $(\gamma$ and $\zeta$ are normalized to zero and 1 for the first indicator for identification purposes), and $v_{q k}$ is the normally distributed error term with zero mean and standard deviation $\sigma_{v}$.

The distributions of the latent variable and the indicators are respectively: 


$$
\begin{aligned}
& f_{L V}\left(L V_{q n} \mid S E ; \alpha, \sigma_{\omega}\right)=\frac{1}{\sigma_{\omega}} \phi\left(\frac{L V_{q n}-\left(C_{n}+\boldsymbol{\alpha}_{n} \cdot \boldsymbol{S} \boldsymbol{E}_{q}^{\prime}+\eta_{q}\right)}{\sigma_{\omega}}\right) \\
& f_{I}\left(I_{q n k} \mid L V_{q n} ; \gamma, \zeta, \sigma_{\nu}\right)=\frac{1}{\sigma_{v_{n k}}} \phi\left(\frac{I_{q n k}-\gamma_{k}-\zeta_{k}\left(L V_{q n}\right)}{\sigma_{v_{k}}}\right)
\end{aligned}
$$

The probability that individual $q$ will choose alternative $\mathrm{j}$ is given by:

$$
P_{q j}=\int_{\omega} \int_{\eta} P_{q j}\left(L V_{q n}\left(\omega_{q}, \eta_{q}\right)\right) \prod_{n} f_{L V_{q n}}\left(\eta_{q}\right) \prod_{k} f_{I_{q u k}}\left(L V_{q n}\left(\omega_{q}, \eta_{q}\right)\right) f(\omega) f(\eta) d \omega d \eta
$$

Models are estimated using PythonBiogeme (Bierlaire and Fetiarison, 2009).

\section{Estimation results}

Tables 4 and 5 present the results of the final hybrid model $^{* *}$, estimated separately for the estimation and validation samples. To test whether disregarding the serial-correlation effect influenced the estimation results, both the models estimated without serial-correlation (first 2 models in Tables 4 and 5) and including serial-correlation (last column in both tables) are shown. Table 4 gives the results for the binary logit model, Table 5 those for the indicators of latent variables.

First of all we estimated the discrete and latent models (one for each latent variable) separately. Having identified the best discrete model specification and the three best latent models, the hybrid choice model was then estimated simultaneously. The model presented in Tables 4 and 5 includes only two latent variables "perception of bicycle as a means of transport" and "perception of context characteristics", as the third, "perception of bikeability", was significant only when estimated alone; it was not identified when estimated together with the other two. This result is not surprising because the factor analysis also revealed this factor to be below the reliability threshold.

As far as the discrete part of the model is concerned, several socioeconomic characteristics have a direct influence on the propensity to cycle. In particular, men and younger people are more likely to cycle than women and older persons respectively (the age variable is included in the specification as continuous, so the propensity to cycle decreases as age increases). Most studies conclude that men cycle more than women (Heinen et al., 2010), but this tendency seems to be related to cycling culture: Garrard et al. (2008) found that in countries with low rates of cycling males are more likely to cycle than females; by

\footnotetext{
${ }^{* *}$ The variables described in Section 4 were all tested in the model specification, retaining only the significant ones. All continuous variables were also tested as dummy variables for different ranges to test for non-linear effects.
} 
contrast, in countries with high cycling rates, such as the Netherlands and Denmark, cycling is more evenly spread across the two genders (Heinen et al., 2013). A likely explanation, in this case study, is that women usually have more family responsibilities, such as shopping and picking up children, activities that are less likely to be undertaken by bike. This is confirmed by the negative sign of the presence of children in the household (dummy variable that takes value 1 if children are present, 0 otherwise). Indeed, families with children are less likely to cycle. By contrast, in the existing literature the relation between cycling and age is not so clear. Some researchers indicate that cycling levels decline with age (Dill \& Voros 2007; Pucher et al. 1999) while others (Wardman et al., 2007; De Geus, 2007) have found that age is not a significant factor. In the context of this study, the age-based result probably depends on the fact that the majority of young adults have low incomes and cannot afford more expensive travel mode options. Indeed, individuals with greater purchasing power appear to be less likely to cycle. On the other hand the relationship between cycling and income reported in the literature is ambiguous. While some workers (Stinson \& Bhat, 2005; Dill \& Voros, 2007) report a positive relationship between income and cycle use, Parkin et al. (2008) found that in England there is a link between lower incomes and lower bicycle share.

Further, people who are informed about bike sharing are more likely to cycle (and of course yearly subscribers more than non-subscribers), than the uninformed, confirming that the motivation to cycle positively influences the propensity to cycle.

The two latent variables have positive sign, indicating that increased perception of the context (i.e. the availability of cycling infrastructure and facilities), increases the propensity to cycle (positive sign of the latent variable). The presence of cycling facilities is a significant factor in the choice to cycle. Further, almost all earlier studies have revealed that the existence of a bike network could increase the propensity to cycle for utilitarian purposes (Hunt \& Abraham, 2007; Dill \& Voros, 2007).

The same can be said of the perception of the bicycle as a means of transport (the latent variable has a positive sign). Regarding the magnitude of the estimates of the two latent variables, the need for cycling infrastructure and facilities affects the propensity to cycle to a greater extent than the perception of the bicycle as a means of transport (1.53 vs. 0.98). In other words, under the same perception of the context, a person who perceives the bicycle as a means of transport is more likely to cycle.

The latent variables are also included in the interaction with socioeconomic variables. Particularly, the interaction between the perception of bicycle as a means of transport and the number of household members is negative and highly significant. This indicates that the effect of the perception of the bicycle as a means of transport is positive but less so for larger households. This is not surprising, as it could be related to the responsibilities of households with children where many activities (pickup and drop off, shopping, etc.) are difficult to do by bike.

Another significant interaction is between the latent variable perception of the context and the number of cars per household. This interaction can be interpreted similarly to the above (negative sign of the 
parameter). As the number of available cars increases, so the effect of the perception of the context on the propensity to cycle decreases. This may be because in multi-car households members are less concerned about the context characteristics for cycling. This result is in agreement with other studies in which car availability is widely reported as negatively related to bicycle choice (Muñoz et al., 2016).

As far as the latent part of the model is concerned, the socioeconomic variables that contribute to specifying the latent variable "perception of context" are: high level of education, low-medium income, car availability, a certain number of bikes in the household and no children. This is easily understood as those who are more likely to cycle (number of bikes in the household, absence of family impediments, low-medium income) assign importance to the availability of cycling facilities. In this context, $90.5 \%$ of the sample own a car, so this variable does not single out a certain target. For "perception of bicycle as a means of transport", the socioeconomic variables defining the latent variable indicate that a high level of education and bike ownership correspond to high perception of the benefits of cycling.

We estimated the validation model (550 obs.) using the same specification as above, apart from the interactions of latent variables with socioeconomic variables, as the sample size was too small to allow identification. The validation results indicate that the model performs well with the hold-out sample. All the coefficients estimated are significant at $95 \%$, with only 2 exceptions. More importantly, the coefficients do not differ significantly from those estimated with the estimation sample. Furthermore, in order to verify model performance, we simulated the choice probabilities using the two models estimated for the estimation and validation samples. As illustrated in Table 6, the two models perform similarly, in particular the probability to cycle is practically identical ( $49 \% v s .49 .7 \%)$ for both models.

Finally note that in the model that takes into account serial correlation, the coefficients do not differ significantly from those estimated in the model that does not. However, standard deviation that accounts for serial correlation is highly significant, suggesting it cannot be disregarded. 
Table 4: Model results

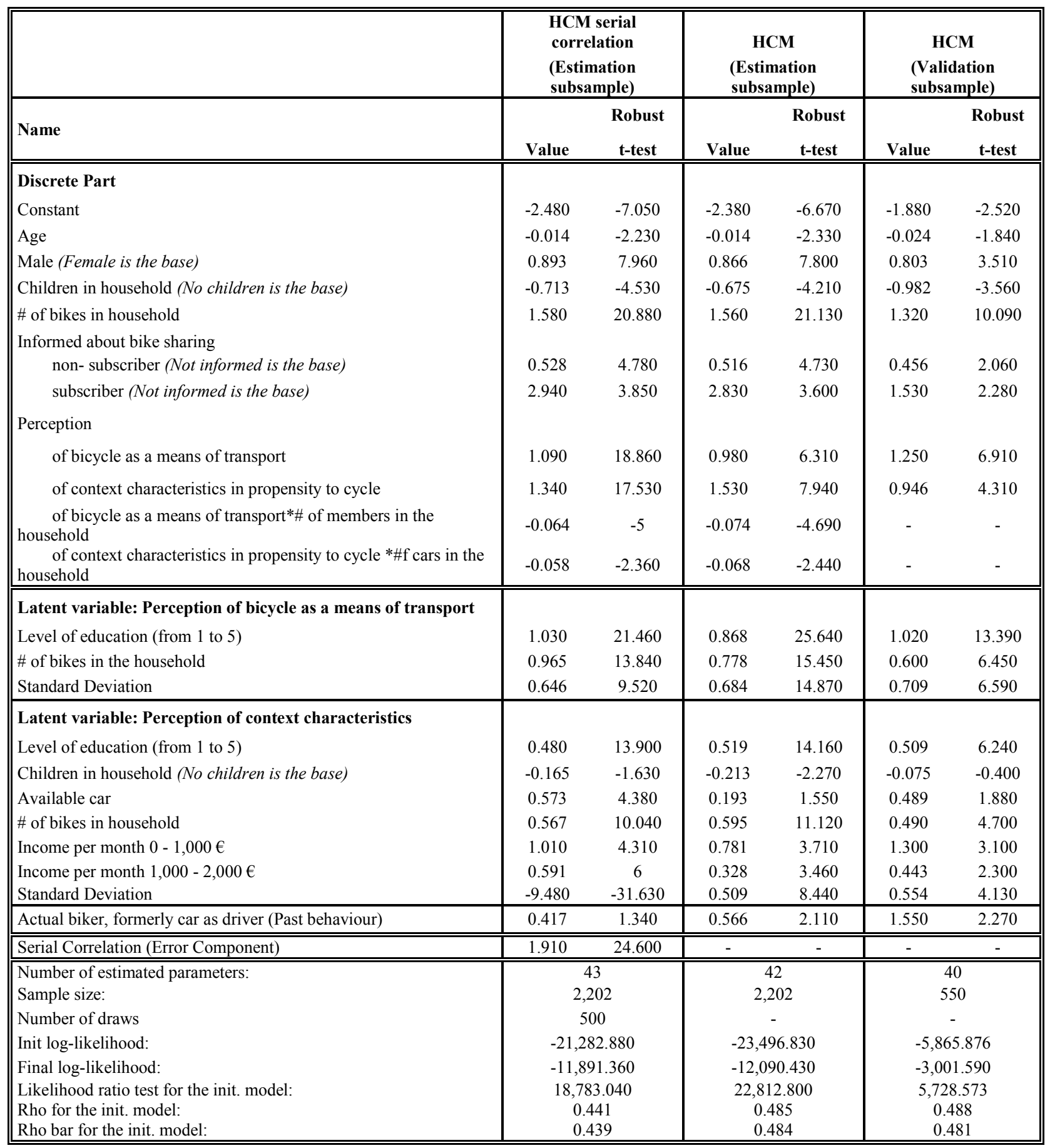




\section{Conclusions}

Experience gained in utility cycling in major European cities has shown a correlation between travel mode choice in urban environments and measures that encourage or hinder the use of a given mode. This is particularly true of the bicycle, a marginal and not generally recognised form of transport for utilitarian purposes, precisely because much of the existing infrastructure has been designed for use preferentially by other means of transport. Indeed, in many contexts, such as the case at hand, where cycling culture does not exist, the bicycle may not be perceived as an alternative means of travel, in the same way as the private car and public transport. Thus the decision to cycle entails a more radical change compared to the switch between traditional travel modes.

For this reason it is essential, when implementing measures for increasing bike commuting, to identify and understand those factors influencing travel choice, in order to explain why certain people choose to bike commute whereas others prefer to travel by traditional means of transport. This will enable planners to develop comprehensive mobility systems, radically different from existing ones. In particular, exploring travel behaviour and mobility styles of potential and existing cyclists serve as the basis for formulating programmes that aim to change commuter preferences in urban mobility choices.

In this work we studied the role of perception in the propensity to commute by bike. In particular we focused on three aspects of perception: bike as a means of transport, bikeability (in terms of usefulness and safety) and bike infrastructure. The context of application concerns utility cycling by public employees in Cagliari (Italy). To measure the impact of individuals' perception on the probability to cycle we estimated hybrid choice models that account for serial correlation between error terms in the discrete and latent perceptions. We also validated the model results, using a hold-out sample. The results suggest that, beside individual characteristics (young adults, males, with no children in the household are more willing to cycle), the perception of the context characteristics (i.e. the availability of cycling infrastructure and facilities) and of the bicycle as a means of transport (benefits of cycling) affect the propensity to cycle. Particularly the results show, on the one hand, that the lack of proper infrastructure and facilities create a strong barrier to bicycle use, and on the other that the greater propensity to cycle is directly correlated with the perception of the bike as a means of transport. Indeed, for the same context characteristics, individuals who perceive the bike as a mode of travel are more likely to cycle. Thus, also in view of the large sample size that made it possible to obtain a validation sample and hence robust results, the model results provide scientific evidence, often disregarded, of the importance not only to eliminate barriers through the creation of dedicated infrastructure and facilities, but also to raise the awareness of the bicycle as an alternative commute mode. This is especially true of Italy where the bicycle is perceived more as a form of exercise, or for leisure than as a means of transport. This result provides a useful tool for establishing preliminary policy directions for increasing the propensity to commute by bike. These need to account for individuals' intrinsic characteristics through the provision of information aimed at promoting bicycle use.

Thus, arguably, policies for promoting the bicycle as an alternative mode of transport to motorized vehicles need to combine hard and soft measures. 


\section{Acknowledgements}

This research was sponsored by the Sardinian Regional Government.

\section{References}

Akar, G. \& Clifton, K.J. (2009). The influence of individual perceptions and bicycle infrastructure on the decision to bike. In: Proceedings of Transport Research Board Annual Meeting, Washington DC.

Bamberg, S., \& Schmidt, P. (1994). Car or Bicycle-an Empirical-Test of a Utility-Theory-Approach for Predicting the Choice between Means of Transportation. Kolner Zeitschrift fur Soziologie und Sozialpsychologie, 46(1), pp. 80-102.

Bierlaire, M. (2016). Estimating choice models with latent variables with PythonBiogeme (No. EPFLREPORT-221361).

Bierlaire, M., \& M. Fetiarison (2009). Estimation of Discrete Choice Models: Extending BIOGEME. 9th Swiss Transport Research Conference, Monte Veritá. Switzerland.

Broach, J., Dill, J., \& Gliebe, J. (2012). Where do cyclists ride? A route choice model developed with revealed preference GPS data. Transportation Research Part A: Policy and Practice, 46(10), pp. 17301740 .

Calvey, J.C., Shackleton, J. P., Taylor, M.D., \& Llewellyn, R. (2015). Engineering condition assessment of cycling infrastructure: Cyclists' perceptions of satisfaction and comfort. Transportation Research Part A: Policy and Practice, 78, pp. 134-143.

Carstensen, T.A., \& Ebert, A.K. (2012). Cycling cultures in northern Europe: from 'golden age' to 'renaissance'. Cycling and Sustainability, pp. 23-58.

Cattell, R.B. (1966). The scree test for the number of factors. Multivariate Behavioral Research, 1,pp. 245-276.

Chataway, E.S., Kaplan, S., Nielsen, T.A.S., \& Prato, C.G. (2014). Safety perceptions and reported behavior related to cycling in mixed traffic: A comparison between Brisbane and Copenhagen. Transportation Research Part F: Traffic Psychology And Behaviour, 23, pp. 32-43.

Cherchi, E. \& Cirillo, C. (2010) Validation and forecasts in models estimated from multi-days travel survey. Transportation Research Record 2175(1), pp. 57-64.

De Geus, B. (2007). Cycling to work: psychosocial and environmental factors associated with cycling and the effect of cycling on fitness and health indexes in an untrained working population. Vrije Universiteit, Department of human physiology and sports medicine.

Dickinson, J.E., Kingham, S., Copsey, S., \& Hougie, D.J.P. (2003). Employer travel plans, cycling and gender: will travel plan measures improve the outlook for cycling to work in the UK?. Transportation Research Part D: Transport and Environment, 8(1), pp. 53-67. 
Dill, J., \& Voros, K. (2007). Factors affecting bicycling demand: initial survey findings from the Portland, Oregon, region. Transportation Research Record: Journal of the Transportation Research Board, (2031), pp. 9-17.

Emond, C.R., \& Handy, S.L. (2012). Factors associated with bicycling to high school: insights from Davis, CA. Journal of Transport Geography, 20(1), pp. 71-79.

Fernández-Heredia, Á., Monzón, A., \& Jara-Díaz, S. (2014). Understanding cyclists' perceptions, keys for a successful bicycle promotion. Transportation Research Part A: Policy and Practice, 63, pp. 1-11.

Garrard, J., Rose, G., \& Lo, S. K. (2008). Promoting transportation cycling for women: the role of bicycle infrastructure. Preventive medicine, 46(1), pp. 55-59.

Gatersleben, B., \& Appleton, K.M. (2007). Contemplating cycling to work: Attitudes and perceptions in different stages of change. Transportation Research Part A: Policy and Practice, 41(4), pp. 302-312.

Habib, K.N., Mann, J., Mahmoud, M., \& Weiss, A. (2014). Synopsis of bicycle demand in the City of Toronto: Investigating the effects of perception, consciousness and comfortability on the purpose of biking and bike ownership. Transportation Research Part A: Policy And Practice, 70, pp. 67-80.

Heinen, E., Van Wee, B., \& Maat, K. (2010). Commuting by bicycle: an overview of the literature. Transport reviews, 30(1), 59-96.

Heinen, E., Maat, K., \& Van Wee, B. (2011). The role of attitudes toward characteristics of bicycle commuting on the choice to cycle to work over various distances. Transportation Research Part D: Transport and Environment, 16 (2), pp. 102-109.

Heinen, E., Maat, K., \& van Wee, B. (2013). The effect of work-related factors on the bicycle commute mode choice in the Netherlands. Transportation, 40(1), pp. 23-43.

Hunecke, M., Blöbaum, A., Matthies, E., \& Höger, R. (2001). Responsibility and environment ecological norm orientation and external factors in the domain of travel mode choice behavior. Environment and Behavior, 33 (6), 830-852.

Hunt, J., \& Abraham, J. (2007). Influences on bicycle use. Transportation 34, pp. 453-470.

Kamargianni, M., \& Polydoropoulou, A. (2013). Hybrid choice model to investigate effects of teenagers' attitudes toward walking and cycling on mode choice behavior. Transportation Research Record: Journal of the Transportation Research Board, (2382), pp. 151-161.

Kaplan, S., Manca, F., Nielsen, T. A. S., \& Prato, C. G. (2015). Intentions to use bike-sharing for holiday cycling: An application of the Theory of Planned Behavior. Tourism Management, 47, pp. 34-46.

Kingham, S., Dickinson, J., \& Copsey, S. (2001). Travelling to work: will people move out of their cars. Transport Policy, 8(2), pp. 151-160.

Klapper, D., Ebling, C., Temme, J., 2005. Another look at loss aversion in brand choice data: can we characterize the loss averse consumer? Int. J. Res. Mark. 22, pp. 239-254.

Krizek, K.J. (2012). Cycling, Urban Form and Cities: What Do We Know and How Should We Respond? Cycling and Sustainability, 1, pp. 111-130.

La Paix, L., Cherchi, E. \& Geurs, K. (2015) Integration of unobserved effects in access/egress mode choice models. NECTAR Conference, Chicago, USA. 
Li, Z., Wang, W., Yang, C., \& Ragland, D. R. (2013). Bicycle commuting market analysis using attitudinal market segmentation approach. Transportation Research Part A: Policy and Practice, 47, 56-68.

Ma, L., Dill, J., \& Mohr, C. (2014). The objective versus the perceived environment: what matters for bicycling?. Transportation, 41(6), pp. 1135-1152.

Mabit, S., Cherchi, E., Jensen, A., \& Jordal-Jørgensen, J. (2015) The effect of attitudes on loss aversion: estimation and validation for the case of alternative-fuel vehicles. Transportation Research Part A: Policy and Practice, 82(1), pp.17-28.

Majumdar, B.B., Mitra, S., \& Pareekh, P. (2015). Methodological framework to obtain key factors influencing choice of bicycle as a mode. Transportation Research Record: Journal of the Transportation Research Board, 2512, pp. 110-124.

Maldonado-Hinarejos, R., Sivakumar, A., \& Polak, J.W. (2014). Exploring the role of individual attitudes and perceptions in predicting the demand for cycling: a hybrid choice modelling approach. Transportation, 41(6), pp. 1287-1304.

Meloni, I., Sanjust, B., Sottile, E. (2015). Modeling the propensity to cycle. An experimental analysis. In: World Conference on Transport Research - WCTR 2016 Shanghai. 10-15 July 2016.

Muñoz, B., Monzon, A., \& López, E. (2016). Transition to a cyclable city: Latent variables affecting bicycle commuting. Transportation Research Part A: Policy and Practice, 84, pp. 4-17.

Motoaki, Y., \& Daziano, R.A. (2015). A hybrid-choice latent-class model for the analysis of the effects of weather on cycling demand. Transportation Research Part A: Policy and Practice, 75, pp. 217-230.

Parkin, J., Wardman, M., \& Page, M. (2008). Estimation of the determinants of bicycle mode share for the journey to work using census data. Transportation, 35(1), pp. 93-109.

Pooley, C., Horton, D., Scheldeman, G., Tight, M., Harwatt, H., Jopson, A., \& Mullen, C. (2012). The role of walking and cycling in reducing the impacts of climate change. Transport and Climate Change, 2, pp. 175.

Pucher, J., \& Buehler, R. (2006). Why Canadians cycle more than Americans: a comparative analysis of bicycling trends and policies. Transport Policy, 13(3), pp. 265-279.

Pucher, J., Dill, J., \& Handy, S. (2010). Infrastructure, programs, and policies to increase bicycling: an international review. Preventive Medicine, 50, pp. 106-125.

Pucher, J., Komanoff, C., \& Schimek, P. (1999). Bicycling renaissance in North America?: Recent trends and alternative policies to promote bicycling. Transportation Research Part A: Policy and Practice, 33(7), pp. 625-654.

Sigurdardottir, S. B., Kaplan, S., Møller, M., \& Teasdale, T. W. (2013). Understanding adolescents' intentions to commute by car or bicycle as adults. Transportation research part D: transport and environment, 24, pp. 1-9.

Stinson, M. A., \& Bhat, C. R. (2005, January). A comparison of the route preferences of experienced and inexperienced bicycle commuters. In TRB 84th Annual Meeting Compendium of Papers (No. 051434). 
Van Hout Kurt, 2008. Literature search bicycle use and influencing factors in Europe, Universiteit Hasselt - Instituut voor mobiliteit.

Vandenbulcke, G., Dujardin, C., Thomas, I., de Geus, B., Degraeuwe, B., Meeusen, R., \& Panis, L.I. (2011). Cycle commuting in Belgium: spatial determinants and 're-cycling'strategies. Transportation Research Part A: Policy and Practice, 45(2), pp. 118-137.

Wardman, M., Tight, M., \& Page, M. (2007). Factors influencing the propensity to cycle to work. Transportation Research Part A: Policy and Practice, 41(4), pp. 339-350.

Willis, J. R. (2015). Effect of Recycled Materials on Pavement Life-cycle Assessment: A Case Study. In Transportation Research Board 94th Annual Meeting (No. 15-4109). 